\title{
Instantaneous Frequency Estimation of Multi-Component Non- Stationary Signals using Fourier Bessel series and Time-Varying Auto Regressive Model
}

\author{
G. Ravi Shankar Reddy and Rameshwar Rao
}

\begin{abstract}
In this paper, we propose a novel technique for Instantaneous Frequency (IF) estimation of multi component non stationary signals using Fourier Bessel Series and TimeVarying Auto Regressive (FB-TVAR) model. In the proposed technique, the Fourier-Bessel (FB) expansion decomposes the multi-component non stationary signal into a number of monocomponent signals and TVAR model is used to model each mono-component signal. In TVAR modeling approach the time varying parameters are expanded as a linear combination of basis functions. In this paper, the TVAR parameters are expanded by a discrete cosine basis functions. The maximum likelihood estimation algorithm for model order selection in TVAR models is also discussed. The Instantaneous Frequency (IF) is extracted from the time-varying parameters by calculating the angles of the estimation error filter polynomial roots. The estimation of the TVAR parameters of a multicomponent signal requires the inversion of a large covariance matrix, while the projected technique (FB-TVAR) requires the inversion of a number of comparatively small covariance matrices with better numerical stability properties. Simulation results are presented for Multi component discrete Amplitude and Frequency modulated (AM-FM) signal
\end{abstract}

Keywords-basis function, Fourier-Bessel expansion, Instantaneous Frequency, multi component non stationary signal, Time-varying Auto Regressive model

\section{INTRODUCTION}

$\mathbf{T}$ HE majority of real-life signals are non-stationary and often multi-component. Bat signal, sonar signal, seismic signals, whale sounds, bird songs, musical recordings are some examples of this kind of signals. Moreover, multicomponent non-stationary signals are encountered in various man made signals such as radar, sonar, biomedical engineering and automotive emission. These signals also exist in multiple-signal environments [1].

A multi-component non-stationary signal is often expressed as a sum of individual mono-component signals, where each component has time-variant amplitude and frequency (or) Phase functions. The analysis of such signals involves estimation of instantaneous frequency (IF) function of each mono-component [1]

The spectrogram, the most commonly used TFD, describes the IF laws-IF trajectories in the time-frequency plane of the individual components of the observed multi-component

G. Ravi Shankar Reddy is with dept of ECE, CVR College of Engineering, Hyderabad, India (email: ravigosula_ece39@yahoo.co.in).

R. Rao is with Jawaharlal Nehru Technological University Hyderabad, India (email: rameshwar_rao@hotmail.com). signal. However, its time-frequency resolution, especially for closely spaced components, is often poor. The Wigner-Ville Distribution (WVD), another commonly used TFD, presents high amplitude cross terms for such signals. The ChoiWilliams Distribution has a tradeoff between cross term suppression and time-frequency resolution. In general, a TFD used in analysis of multi-component non-stationary signals should be cross terms free and should have high resolution in the time-frequency domain.[1-5]

Spectral analysis of non-stationary signals, with high frequency-resolution is obtained by using the Time Varying Autoregressive (TVAR) Model [6].In the modeling of nonstationary signals by TVAR process, estimation of the TVAR parameters can be classified into one of the two categories as the adaptive technique and the basis function approach[7].The most popular approach to estimate time varying coefficients is to employ an adaptive algorithm and track the parameters. These methods work well with slowly varying signals but fail to track rapid variation. If the coefficients change fast enough, compared to the algorithm's convergence time, the adaptive algorithm will not be able to track the time varying parameters. Another common approach to estimate the parameters is to expand each time varying coefficient into a set of basis functions. If each coefficients time-evolution can be approximated by a combination of a small number of basis functions, then the estimation task is equivalent to the estimation of the weighting constants in this expansion[6,7].

In this basis function expansion, two issues need to be resolved. First, a general class of basis functions is to be chosen, and then, the significant basis functions need to be selected. Several classes of functions have been proposed including polynomial, wavelet and prolate spheroidal functions. However, no uniform rule exists to indicate which class should be adopted. Moreover, the approach of choosing the significant basis functions is based on trial and error[12].

Non stationary signal is modeled using TVAR process of order $\mathrm{p}$. In the process of modeling, the estimation of the TVAR parameters require the inversion of a covariance matrix of size $\mathrm{p}(\mathrm{q}+1) \times \mathrm{p}(\mathrm{q}+1)$, where $\mathrm{q}$ is the required number of basis functions to represent each TVAR parameter. For modeling multi-component non stationary signal using TVAR process, large number of basis functions (q) and high model order (p) are essential to estimate TVAR parameter, which requires the inversion of a large covariance matrix [12]. 
It is therefore suggested that in such cases of modeling by a TVAR process it would be wiser to perform a component separation before doing estimation of the process parameters. This enables us to decompose a complex estimation problem into a set of sub problems, each much simpler and more favorable from a numerical-computation point of view.

In this paper, we develop a method of signal analysis based on the Fourier-Bessel (FB) expansion and the TVAR process. The multi-component Non stationary signal has been expanded into the FB series. Each component has non overlapping cluster of FB coefficients. The components can be reconstructed directly from the real FB coefficients of the separated clusters. Then, we model each reconstructed component by a lower order TVAR process. In this way it requires the inversion of a number of relatively small correlation matrices with better numerical stability properties.

The paper is organized as follows. In section II it explains the Fourier-Bessel Expansion. It explains the Time-varying Autoregressive modeling in section III. In section IV it explains the selection of basis function and TVAR model order determination by means of Maximum likelihood estimator. In section $\mathrm{V}$ it gives the steps for proposed algorithm. The investigational results of analysis of AM-FM signal is presented in section VI. Concluding remarks are given in section VII.

\section{FOURIER-BESSEL EXPANSION}

Fourier-Bessel expansions decompose the multi component signal in to mono component Signal without any prior information about the frequency band of the signal.

Fourier-Bessel (FB) series is a particular kind of generalized Fourier series (is an infinite series expansion on a finite interval) based on Bessel functions. Consider discrete time signal $x(n)$ over an interval $(0, \mathrm{~N})$ the zero-order $\mathrm{FB}$ series expansion of this signal is expressed as [9]

$$
x(n)=\sum_{m=1}^{M} C_{m} J_{0}\left(\frac{\lambda_{m}}{N} n\right)
$$

The FB coefficients $C_{m}$ are computed by using the relation

$$
C_{m}=\frac{2 \sum_{n=0}^{N} n x(n) J_{0}\left(\frac{\lambda_{m}}{N} n\right)}{N^{2}\left[J_{1}\left(\lambda_{m}\right)\right]^{2}}
$$

Where $\mathrm{M}$ is the order of the FB expansion and $J_{0}$ (.), $J_{1}$ (.) are the zero-order of the Bessel function of the first kind and the first-order of the Bessel function of the first kind, respectively and can be used as basis functions for FB series[9]. $\left\{\lambda_{m} ; \mathrm{m}=1,2, \ldots \ldots ., \mathrm{M}\right\}$ are the ascending order positive roots of $J_{0}(\lambda)=0$

It has been demonstrated in $[9,12]$ that if the center frequency and the bandwidth of a test signal are varied then the order and range of nonzero coefficients of the FB series expansion of the signal are also changed .In particular, it is shown that the order increases with higher center frequency and range widens with larger bandwidth. An explanation of the above properties of the FB series expansion can be obtained by analytically deriving the coefficient $C_{m}$ for the signal

$$
x(n)=\cos (\omega n)
$$

The FB coefficient $C_{m}$ for the above is given by [10]

$$
\begin{aligned}
& C_{m}=\frac{2 \lambda_{m} \cos (\omega N-\alpha)}{J_{1}\left(\lambda_{m}\right)\left[\left(\lambda_{m}^{2}-\omega^{2} N^{2}\right)^{2}+\omega^{2} N^{2}\right]^{1 / 2}} \\
& \text { Where } \alpha=\sin ^{-1}\left(\frac{\omega N}{\left[\left(\lambda_{m}^{2}-\omega^{2} N^{2}\right)^{2}+\omega^{2} N^{2}\right]^{1 / 2}}\right)
\end{aligned}
$$

From (4) It can be verified that the peak value of $C_{m}$ is attained for the order $\mathrm{m}$ where the root $\lambda_{m} \approx \omega N$ and the peak value is given by

$$
C_{m, \text { peak }} \approx \frac{\sin (\omega N)}{J_{1}(\omega N)}
$$

The magnitude of $C_{m}$ decreases quickly away from the order where the peak value is taking place and the value becomes irrelevant at far-away orders. It should be noted that like the Fourier series coefficients are unique for a given signal, the FB series coefficients $C_{m}$ are also unique for a given signal. Nevertheless, unlike the sinusoidal basis functions in the Fourier series, the Bessel functions are having damped sinusoidal function and will decay over time. This characteristic of the Bessel functions makes the FB series expansion appropriate for non-stationary signals [9, $12]$.

\section{A. Spectrum Representation}

Similar to the Fourier expansion, the FB expansion can also be used for spectrum representation. The benefit lies in the fact that unlike a Fourier coefficient which represents only a single frequency, each FB coefficients represents a band of frequencies. Thus only fewer FB coefficients are adequate to represent a band-pass signal compared to the number of Fourier coefficients. For a given sinusoidal signal $x(n)=\cos (\omega n)$ the FB coefficients will have peak at $\lambda_{m} \approx \omega N$. consequently the relation between the order of the FB expansion and the frequency is given by the following equation $[9,12]$

$\lambda_{m}=2 \pi f N$

Where $N$ is the length of the signal, and $f$ is the frequency in $\mathrm{Hz}$.It is obvious from the above that there is one-to-one correspondence between the order (M) of the FB expansion where the coefficients attain peak magnitude and frequency 
content of a signal $[9,10]$. The FB expansion represents the spectrum of a signal like the Fourier expansion. Since the Fourier transform of the Bessel function

$$
\begin{aligned}
& \mathrm{s}(\mathrm{n})=J_{0}\left(\frac{\lambda_{m}}{N} n\right) \text { is given by } \\
& \mathrm{s}(\omega)=\frac{1}{\sqrt{\left(\frac{\lambda_{m}}{N}\right)^{2}-\omega^{2}}} \text { for }|\omega|<\frac{\lambda_{m}}{N}
\end{aligned}
$$

Each term $C_{m} J_{0}\left(\frac{\lambda_{m}}{N} n\right)$ of (1) has an approximate bandwidth $\omega_{B} \cong \frac{\lambda_{m}}{N}$ in the spectral domain. Consequently, the reconstruction of $x(n)$ using the first $M$ terms has a maximum bandwidth of $\omega_{\max } \cong \frac{\lambda_{M}}{N}$.

When $x(n)$ is the sum of $\mathrm{L}$ cosine signals $\cos \left(\omega_{i} \mathrm{n}\right)$ i.e.

$$
x(n)=\sum_{i=1}^{L} \cos \left(\omega_{i} \mathbf{n}\right)
$$

The FB coefficients of the above signal is

$$
C_{m}=\sum_{i=1}^{L} \frac{2 \lambda_{m}}{J_{1}\left(\lambda_{m}\right)}\left(\frac{\cos \left(\omega_{i} N-\alpha_{i}\right)}{\left[\left(\lambda_{m}^{2}-\omega_{i}^{2} N^{2}\right)^{2}+\omega_{i}^{2} N^{2}\right]^{1 / 2}}\right)
$$

Where

$$
\alpha=\sin ^{-1}\left(\frac{\omega_{i} N}{\left[\left(\lambda_{m}^{2}-\omega_{i}^{2} N^{2}\right)^{2}+\omega_{i}^{2} N^{2}\right]^{1 / 2}}\right)
$$

It can be shown that for well-separated frequencies $\omega_{i}$ every expression on the right-hand side of (10) will represent a region of the $\mathrm{FB}$ coefficients where the coefficients are nonzero corresponding to a sub-signal of the composite signal $x(n)$. Since coefficients are real, each cosine components can be directly reconstructed from the FB coefficient plot $[9,11]$

\section{B. Applications of Fourier-Bessel decomposition}

1) One can obtain different band-limited signals by selecting appropriate FB coefficients in re-synthesizing signals. Further by means of each band-limited signal one can perform AM-FM analysis for different speech analysis tasks.

2) For decomposition of a signal into its constituent components, the FB series based technique is beneficial over the technique based on the filter-bank approach, because we do not need any prior information about the frequency band of the signal.

\section{Requisite Conditions for Fourier-Bessel Decomposition}

1) The FB expansion order $M$ must be known a priory. Since the interval between successive zero-crossing of the Bessel function $J_{0}(\lambda)$ increases slowly with time and approaches $\pi$ in the limit. When order $M$ is not known, then for covering full signal bandwidth, that is, the half of the Sampling frequency, $M$ must be equal to the length of the signal

2) Because the FB decomposition basically represents only the oscillatory nature of a signal, the DC component of the signal, if any must be removed prior to the decomposition.

In the present study, we presume that the non stationary signals are well separated in the frequency domain, and the signals will be associated with various different nonoverlapping clusters of the FB coefficients. Consequently, each signal of the multi-component non stationary signal can be reconstructed separately by identifying and separating the corresponding FB coefficients

\section{III.TVAR MODELING}

The non stationary discrete-time stochastic process $x_{n}$ is represented by $\mathrm{p}^{\text {th }}$ order TVAR model as [19]

$x_{n}=-\sum_{k=1}^{p} a_{k, n} x_{n-k}+v_{n}$

Here $a_{k, n}$ are time-varying coefficients and $v_{n}$ is a stationary white noise process with mean is zero and variance is $\sigma_{v}{ }^{2}$. According to the time-varying coefficients evolution, TVAR model is likely to be categorized in to two group's i.e. adaptive method and basis function approach[16,18].

TVAR model based on the basis function technique is able to trace a strong non-stationary signal that is why this model is focused in the present study[19].In this technique, each of its time-varying coefficients are modeled as linear combination of a set of basis functions. The purpose of the basis is to permit fast and smooth time variation of the coefficients. If we denote $u_{m, n}$ as the basis function and consider a set of $(q+1)$ function for a given model, we can state the TVAR coefficients in general as

$a_{k, n}=\sum_{m=0}^{q} a_{k m} u_{m, n}$

In the basis function approach not only the model order $\mathrm{p}$, but also the basis functions $u_{m, n}$, and the expansion dimension $\mathrm{q}$ must be chosen. From (13) we examine that, we have to calculate the set of parameters $a_{k m}$ for $\left\{\mathrm{k}=1,2, \ldots \ldots . ., \mathrm{p} ; \mathrm{m}=0,1,2, \ldots \ldots \ldots . ., \mathrm{q} ; a_{0 m}=1\right\}$ in order to compute the TVAR coefficients $a_{k, n}$, and the TVAR model is absolutely specified by this set. The estimation by the basis function approach is to calculate not the timevarying parameters $a_{k, n}$, but the unknown constant coefficients $a_{k m}$.

The TVAR coefficients are designed as follows, we consider single realization of the process $x_{n}$. For a given realization of $x_{n}$ we can analyze (12) as a time-varying linear 
prediction error filter and consider $v_{n}$ to be the prediction error[19]

$$
v_{n}=x_{n}-\hat{x_{n}}
$$

Where

$$
\hat{x_{n}}=-\sum_{k=1}^{p} a_{k, n} x_{n-k}
$$

The prediction error can be written as

$v_{n}=x_{n}+\sum_{k=1}^{p} \sum_{m=0}^{q} a_{k m} u_{m, n} x_{n-k}$

The total squared prediction error, is now specified by

$$
\begin{aligned}
& \varepsilon_{p}=\sum_{\tau}\left|v_{n}\right|^{2} \\
& \varepsilon_{p}=\sum_{\tau}\left|x_{n}+\sum_{k=1}^{p} \sum_{m=0}^{q} a_{k m} u_{m, n} x_{n-k}\right|^{2}
\end{aligned}
$$

Here, $\tau$ is the interval over which the summation is performed .In covariance technique for modeling the non stationary stochastic process $x_{n}$ we formulate no assumptions on the information outside $[0, \mathrm{~N}-1]$ and set $\tau=[p, N-1]$. The time-varying parameters $a_{\mathrm{km}}$ are estimated by minimizing the mean squared prediction error in (17) can be found by means of setting the gradient of $\epsilon_{p}$ with respect to $a_{l g}^{*}$ zero

$$
\begin{aligned}
& \frac{\partial \varepsilon_{p}}{\partial a_{\mathrm{lg}}^{*}}=\sum_{\tau} \frac{\partial v_{n} v_{n}^{*}}{\partial a_{\mathrm{lg}}^{*}}=\sum_{\tau} v_{n} \frac{\partial v_{n}^{*}}{\partial a_{\mathrm{lg}}^{*}}=0 \\
& \{l=1,2, \cdots, p ; g=0,1, \cdots, q\}
\end{aligned}
$$

Where

$v_{n}^{*}=x_{n}^{*}+\sum_{l=1}^{p} \sum_{g=0}^{q} a_{\lg }^{*} u_{g, n}^{*} x_{n-l}^{*}$

And the derivative of $v_{n}^{*}$ with respect to $a_{l g}^{*}$ is $u_{g, n}^{*} x_{n-l}^{*}$.consequently, (18) become,

$\sum_{\tau} v_{n} u_{g, n}^{*} x_{n-l}^{*}=0$

The above condition is corresponding to the orthogonality law encountered in stationary signal modeling. Substituting for $v_{n}$ in (19) from (16) we have

$\sum_{\tau}\left(x_{n}+\sum_{k=1}^{p} \sum_{m=0}^{q} a_{k m} u_{m, n} x_{n-k}\right) u_{g, n}^{*} x_{n-l}^{*}=0$

Now we define a function $c_{m g}(l, k)$ as shown below,

$c_{m g}(l, k)=\sum_{\tau} u_{m, n} x_{n-k} u_{g, n}^{*} x_{n-l}^{*}$

Using the above definition in (20) we have, $\sum_{k=1}^{p} \sum_{m=0}^{q} a_{k m} c_{m g}(l, k)=-c_{0 g}(l, 0)$

The above equation represents a system of $p(q+1)$ linear equations. The above linear equations can be articulated efficiently in matrix form as follows, we first define a column vector $a_{m}$ as follows

$a_{m}=\left[a_{1 m} a_{2 m} \cdots a_{p m}\right]^{T}$

where

$m=0,1, \cdots, q$

We now use the function in (21) to find the following matrix for $0 \leq(m, g) \leq q$

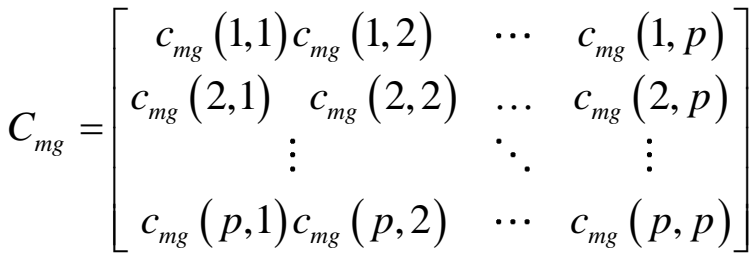

The above matrix is pxp and all the dissimilar values for $\mathrm{m}$ and $g$ result in $(q+1) x(q+1)$ such matrices, by means of these matrices, we can now describe a block matrix as shown below,

$C=\left[\begin{array}{cccc}C_{00} & C_{01} & \cdots & C_{0 q} \\ C_{10} & C_{11} & \ddots & C_{1 q} \\ : & : & : & : \\ \cdot & \cdot & \cdot & \cdot \\ C_{q 0} & C_{q 1} & \cdots & C_{q q}\end{array}\right]$

Matrix $C$ has $(q+1) x(q+1)$ elements and each element is a pxp matrix follow-on in a $\mathrm{C}$ of size $\mathrm{p}(\mathrm{q}+1) \mathrm{x} \mathrm{p}(\mathrm{q}+1)$. Now we describe a column vector $d_{g}$ as shown below

$d_{g}=\left[\begin{array}{ccc}c_{0 g}(1,0) c_{0 g}(2,0) \cdots & c_{0 g}(p, 0)\end{array}\right]^{T}$

where $g=0,1, \cdots, q$

Using the definitions in (23)-(26) we can write down the linear system of equations (22) in matrix form as

$$
\begin{aligned}
& \underbrace{\left[\begin{array}{ccc}
C_{00} & \cdots & C_{0 q} \\
\vdots & \ddots & \vdots \\
C_{q 0} & \cdots & C_{q q}
\end{array}\right]}_{C}\left[\begin{array}{c}
a_{0} \\
\vdots \\
a_{q}
\end{array}\right]=-\left[\begin{array}{c}
d_{0} \\
\vdots \\
d_{q}
\end{array}\right] \\
& C a=-d
\end{aligned}
$$

Note that as soon as $\mathrm{q}=0$, the above equation reduces to the Yule-walker equations (YWE) for a stationary AR model. The set of TVAR parameters $a_{k m}$ are elements of $a$ and can be calculated by solving the above matrix equation. The predictor coefficients $a_{k, n}$ that minimize the prediction error $\epsilon_{p}$ can now be calculated using (13). 


\section{PARAMETER SELECTION}

The TVAR parameter selection is basically depends on three degrees of freedom, such as the TVAR order $p$, the basis function order q, and the set of basis functions $u_{m, n}$.

\section{A. Choice of the Basis Functions}

The basis functions $u_{m, n}$ must be independent and nonzero for $\mathrm{n}=0,1, \ldots . . \mathrm{N}-1$, and $u_{m, n}=1$,if $\mathrm{n}=0$.If a priori information about the signal variation is known, the basis functions should be chosen such that the trends in parameter change is retained. In case, when a priori information is unavailable selection of basis is trial and error[16, 19].

According to equation(13), no particular constraint is imposed on the basis $u_{m, n}$ consequently; one will be able to track only variations which are approximable by this set of functions. Numerous solutions have been projected, in the literature such as time basis functions, Legendre polynomial, Chebyshev polynomial, Discrete prolate spheroidal (DPSS) sequence, Fourier basis, Discrete cosine basis, Walsh basis, Multi wavelet basis, none of these solutions seems to be perfect, since the selection of $u_{m, n}$ desires some priori information upon the time variations present in $x_{n}[16,19]$. Then again, basis such as prolate spheroidal functions are extremely tough to generate. For AM-FM Analysis Discrete cosine basis functions is best suitable basis function [25]

Discrete Cosine basis function

$u_{m, n}=\alpha(\mathrm{m}) \cos \cos \left(\frac{\pi \mathrm{m}(2 \mathrm{n}+1)}{2 \mathrm{~N}}\right)$

Where

$$
\alpha(\mathrm{m})=\left\{\begin{array}{c}
\sqrt{\frac{1}{N}} ; m=0 \\
\sqrt{\frac{2}{N}} ; m=0,1,2 \ldots \ldots q
\end{array}\right.
$$

$$
\mathrm{n}=1,2 \ldots . \mathrm{N}
$$

\section{B. Order Selection}

In the presence of noise The TVAR model can distinguish several time-varying spectral peaks well. However it is sensitive to model order change. False spectral peaks may be produced by the TVAR modeling approach, when an erroneous model order is chosen. Thus, the determination of right model order in TVAR modeling is a significant issue. There are few techniques in choice of TVAR model order. For instance, Bayesian technique [16] and Akaike information criterion (AIC) [17] are used for the determination of model orders in TVAR models. In this article, we consider the choice of the model order as a Maximum-likelihood (ML) estimation [18] technique. In this technique, by maximizing the likelihood function we can determine the model order

\section{Maximum likelihood estimation (MLE)}

The TVAR Model for the non stationary discrete-time stochastic process $x_{n}$ is

$x_{n}=-\sum_{k=1}^{p} \sum_{m=0}^{q} a_{k m} u_{m, n} x_{n-k}+v_{n}$

The above equation can be represented in compact form as

$x_{n}=-Z^{T}[n] a+v_{n}$

Where $Z[n]$ is

$Z[n]=\Phi[n] \otimes u[n]$

Here, $\otimes$ denote Kronecker multiplication

$$
\begin{aligned}
& \Phi[n]=\left[x_{n-1}, x_{n-2} \ldots \ldots x_{n-p}\right]^{T} \\
& u[n]=\left[u_{0 n}, u_{1 n} \ldots \ldots u_{q n}\right]^{T} \\
& a=\left[a_{1}^{T}, a_{2}^{T} \ldots \ldots a_{p}^{T}\right]
\end{aligned}
$$

Here

$a_{k}^{T}=\left[a_{k 0}, a_{k 1} \ldots . . a_{k q}\right]$

Step-1: compute

$$
Z[n]=\Phi[n] \otimes u[n]
$$

Step-2: calculate

$$
C=-\left(\sum_{n=p}^{N} Z[n] Z^{T}[n]\right)^{-1}\left(\sum_{n=p}^{N} Z[n] x[n]\right)
$$

Step-3: Estimate

$$
\hat{\beta}=\frac{1}{N} \sum_{n=0}^{N}\left[x_{n}-C^{T} Z[n]\right]^{2}
$$

Step-4: Obtain the cost function

$$
\begin{aligned}
& J(p, q)=\frac{p(q+1)+2-N}{2} \log (2 \pi \hat{\beta}) \\
& -\frac{1}{2} \log \left|\sum_{n=0}^{N} Z[n] Z^{T}[n]\right|
\end{aligned}
$$

Step-5: Maximize the above cost function to select the expansion dimension $q=q_{o p t}$ and the model order $p=p_{o p t}$ 
Where $p_{\text {opt }} \in\left\{1,2,3,4 \cdots \cdots p_{\max }\right\}$

$$
q_{\text {opt }} \in\left\{0,1,2,3,4 \cdots \cdots q_{\max }\right\}
$$

\section{PRoposed ALGORITHM}

Our procedure based on the FB expansion and the TVAR model for modeling a multi-component non stationary signal requires the following steps:

\section{Step 1: Component separation}

Calculate the FB coefficients $C_{m}$ for a given signal using (2). Every component of the multi-component non stationary signal has non-overlapping cluster of FB coefficients. Since coefficients are real; each component is directly reconstructed by using (1) from coefficient versus order plot. For every mono component signal apply TVAR model

\section{Step 2: TVAR Based IF estimation}

1) Compute TVAR model order $p$ and $q$ using MLE algorithm, choose the basis function $\boldsymbol{U}_{m n}$ $\mathrm{m}=1,2 \ldots \ldots \mathrm{q}, \mathrm{n}=1,2, \ldots \ldots, \mathrm{N}$.

2) For covariance technique of signal modeling set $\tau=[p, N-1]$ and compute $c_{m g}(l, k)$ by means of equation (21) to find the matrix $C_{m g}$ in (24), subsequently, set up the matrix $\mathrm{C}$ in (25), as well, use $c_{m g}(l, k)$ to calculate $d_{g}$ in (26).

3) Calculate the TVAR parameters $a_{k, m}$ by solving $\mathrm{Ca}=-\mathrm{d}$, in (27) and form the coefficients $a_{k, n}$ using (13)

4) Solve the roots of the time-varying autoregressive polynomial formed by TVAR linear prediction filter.

$\mathrm{A}(\mathrm{z} ; \mathrm{n})=1+\sum_{k=1}^{p} a_{k, n} z^{-k}$ at each instant $\mathrm{n}$ to find the time-varying poles: $P_{i, n}, \mathrm{i}=1,2 \ldots \mathrm{p}$

5) The IF of the non stationary signal, for each sample instant $\mathrm{n}$ can be estimated from the instantaneous angles of the poles using $f_{i, n}=\frac{\arg \left[P_{i, n}\right]}{2 \pi}$ for $\left|P_{i, n}\right| \cong 1$

\section{SIMULATION RESULTS}

We have considered a two-component Amplitude modulated and Frequency modulated (AM-FM) signals to study the performance of the proposed FB-TVAR method.

FB Expansion separates multi-component AM-FM signal in to mono-component AM-FM signal, then apply TVAR model to each mono component signal. The Instantaneous frequency (IF) is extracted from the time-varying parameters by calculating the angles of the estimation error filter polynomial roots

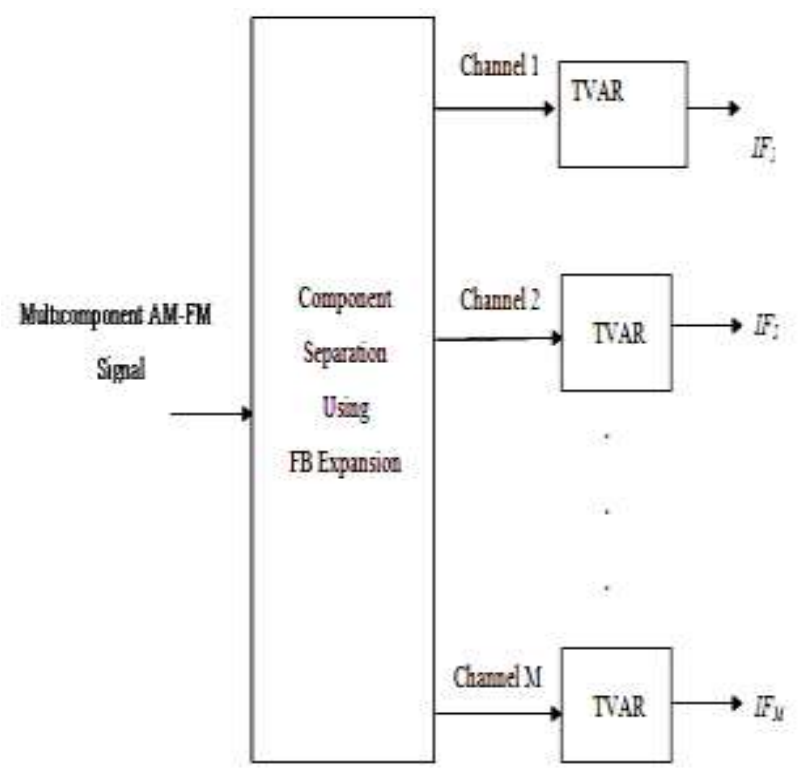

FB-TVAR Method

\section{1) Multi component AM-FM sinusoidal signal}

The sequence $y(n)$ consisting of M single-tone AM-FM sinusoidal signals is represented by [9]

$$
y(n)=\sum_{i=1}^{M} A_{i}\left(1+\mu_{i} \cos \left(v_{a i} n\right)\right) \cos \left(\omega_{i} n+\beta_{i} \sin \left(v_{f i} n\right)\right)
$$

For simulation, the signal consisting of two $(M=2)$ single tone AM-FM signals is sampled at $\mathrm{N}=512$ points. Two sets of parameters in (41) are chosen as

$$
\begin{aligned}
& A_{1}=1, \mu_{1}=0.8, v_{a 1}=2 \pi \times 0.0063, \omega_{1}=2 \pi \times 0.1250 \\
& \beta_{1}=2, v_{f 1}=2 \pi \times 0.0094
\end{aligned}
$$

$A_{2}=1, \mu_{2}=0.6, v_{a 2}=2 \pi \times 0.0125, \omega_{2}=2 \pi \times 0.2500$,

$\beta_{2}=1.5, v_{f 2}=2 \pi \times 0.0125$

IF law for first component signal is given by

$f_{1 n}=\frac{\omega_{1}+\left(\beta_{1} * v_{f 1}\right) \cos \left(v_{f 1} n\right)}{2 \pi}$

IF law for second component signal is given by

$f_{2 n}=\frac{\omega_{2}+\left(\beta_{2} * v_{f 2}\right) \cos \left(v_{f 2} n\right)}{2 \pi}$

The plots of the two-component AM-FM signal, the first component, and second component AM-FM signals are shown in Figs.1-3 respectively. The composite signal is expanded into FB expansion and the plot of FB coefficients for the composite signal is shown in Fig.4.The first 
component of the multi-component signal is separated and reconstructed using 50-190(From Fig.4.) significant nonoverlapping FB coefficients. The reconstructed signal is shown in Fig.5. The second component of the multicomponent signal is separated and reconstructed using 210315 (From Fig.4.) significant non-overlapping FB coefficients. The reconstructed signal is shown in Fig.6.

Each of the two reconstructed AM-FM signals from the clusters of non-overlapped FB coefficients is modeled by TVAR process. By using step 2 in the proposed algorithm we can estimate Instantaneous Frequency (IF) of each reconstructed AM-FM signal.

For separated first component and second component of two component AM-FM signal apply TVAR model with discrete cosine basis. Using Maximum likely hood estimation algorithm we have computed TVAR Model order $\mathrm{p}=2$ and $\mathrm{q}=22$, for first component signal, $\mathrm{p}=2$ and $\mathrm{q}=28$ for second component signal. TVAR coefficients $a_{1, n} a_{2, n}$ for the first component and second component signals are shown in Fig.7.andFig.10.respectively. Trajectory of time varying poles of the first component and second component are shown in Fig.8.and Fig.11.respectively.From Fig.8. and Fig.11.we observe that poles are complex conjugate to each other and also half of the poles are lie in the upper half of Z-plane and remaining half of the poles are lower half of the Z-plane. For IF estimation consider the poles that lie either upper half (or) lower half of the Z-plane.Fig.9.and Fig.12.shows the IF estimation of first component of two component AM-FM signal and the IF estimation of second component of two component AM-FM signal, respectively. From Fig.9.and Fig.12.we observe that estimated IF is close to true IF. The mean square error (MSE) among the true IF and estimated IF for $\mathrm{n}=2,3, \ldots$ , 512 is calculated to be $-118.3282 \mathrm{~dB}$. and $-106.693 \mathrm{~dB}$ respectively.

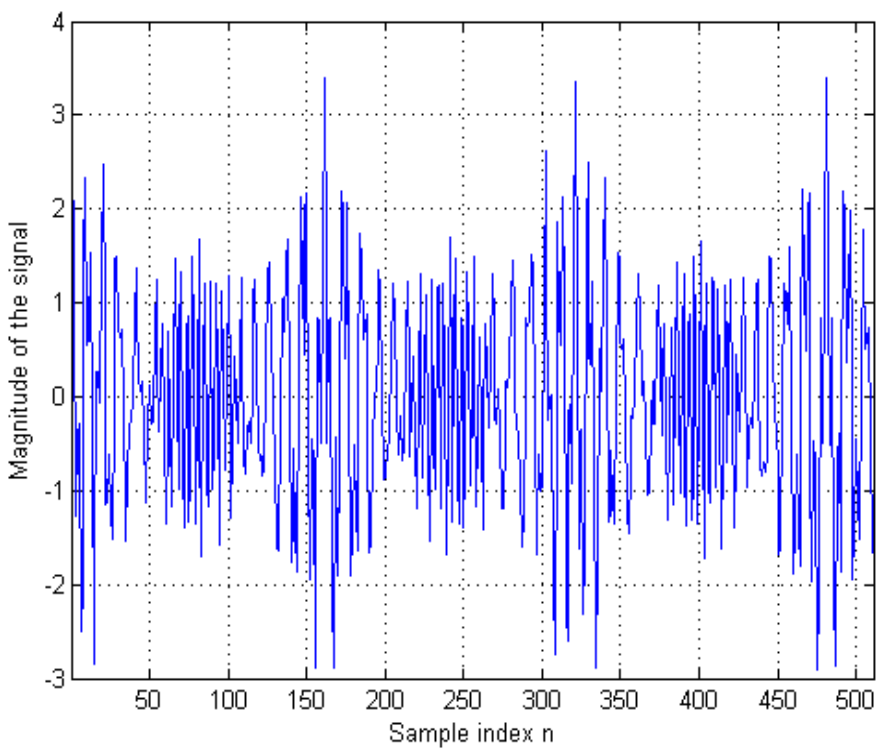

Fig.1.Multi component AM-FM Sinusoidal signal

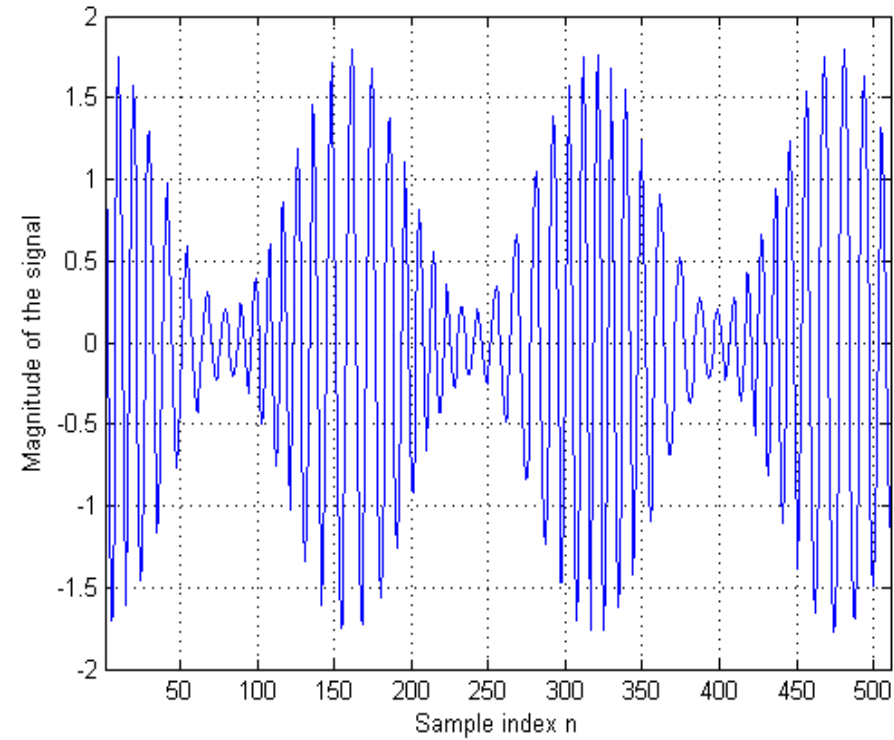

Fig.2. First component of Multi-Component AM-FM Sinusoidal Signal

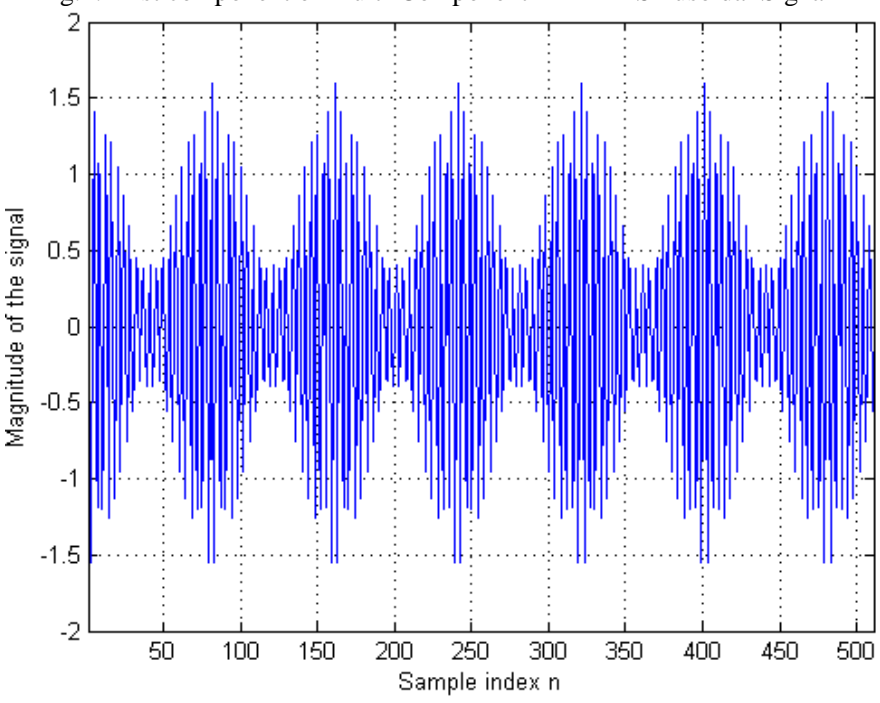

Fig.3.Second-Component of Multi-Component AM-FM Sinusoidal Signal

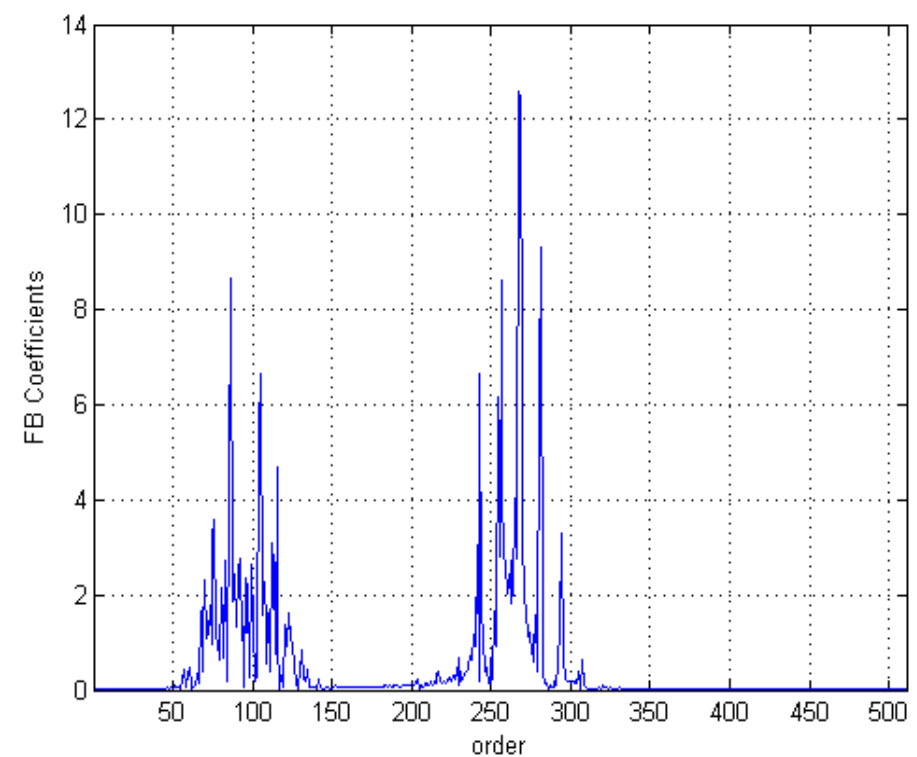

Fig.4.FB Coefficients of the Multi components AM-FM Sinusoidal Signal 


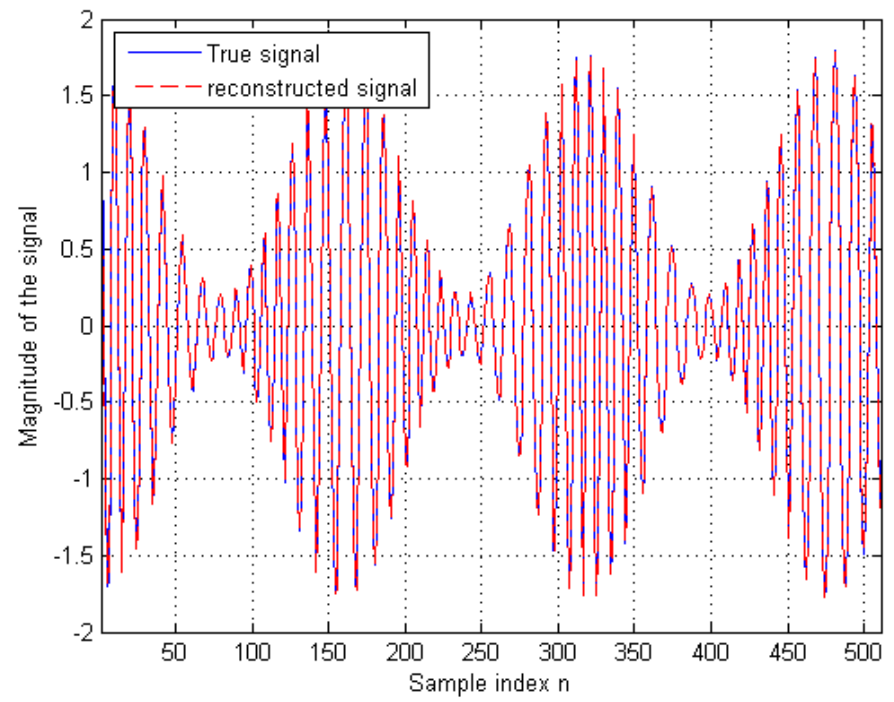

Fig.5.Estimated first component of Multi-Component AM-FM sinusoidal signal using FB expansion

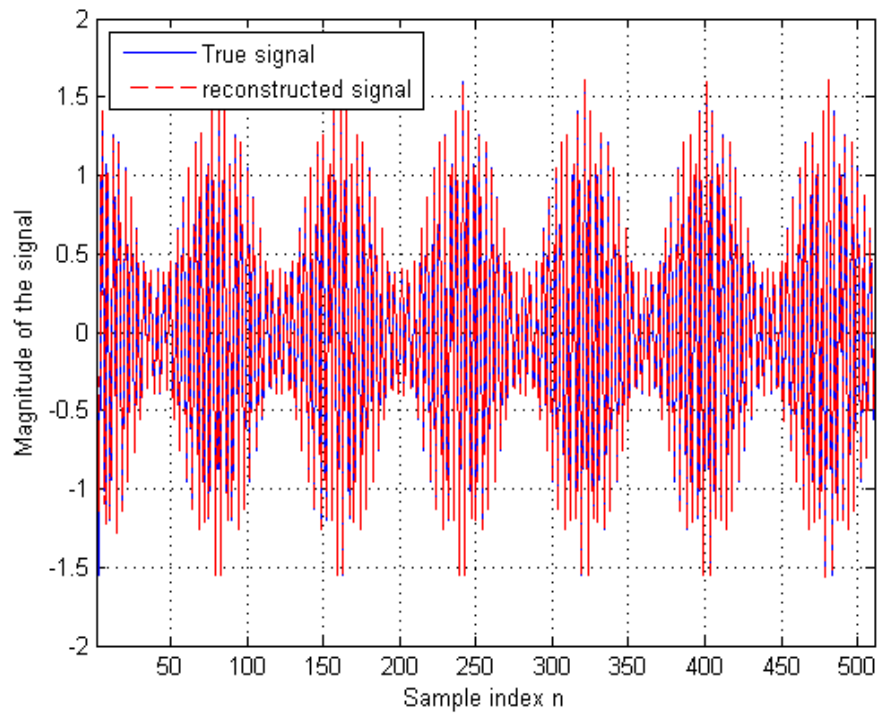

Fig.6.Estimated second component of Multi-Component AM-FM sinusoidal signal using FB expansion
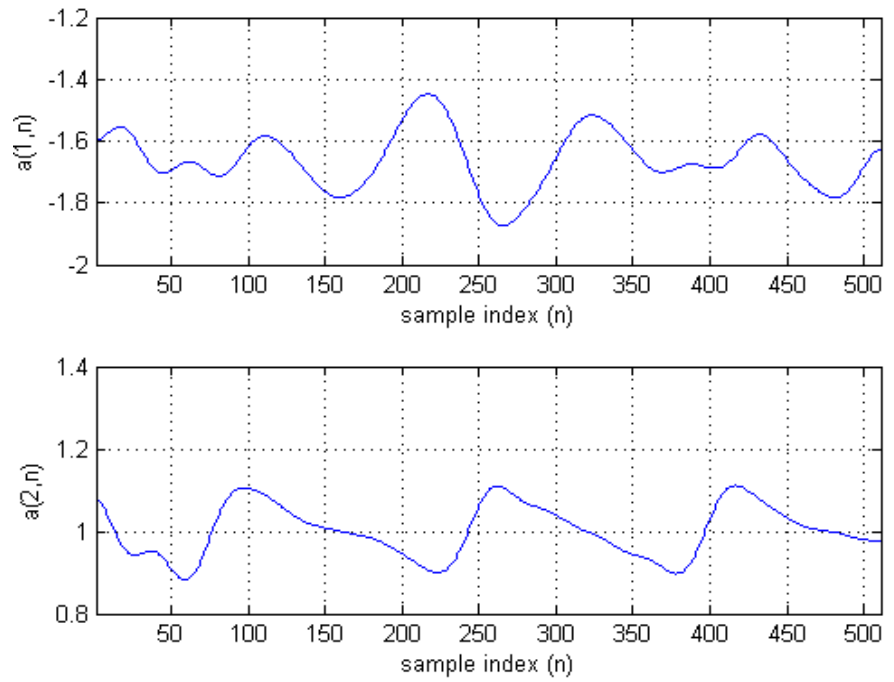

Fig.7.Estimated TVAR Coefficients of first component of Multi-Component AM-FM Signal

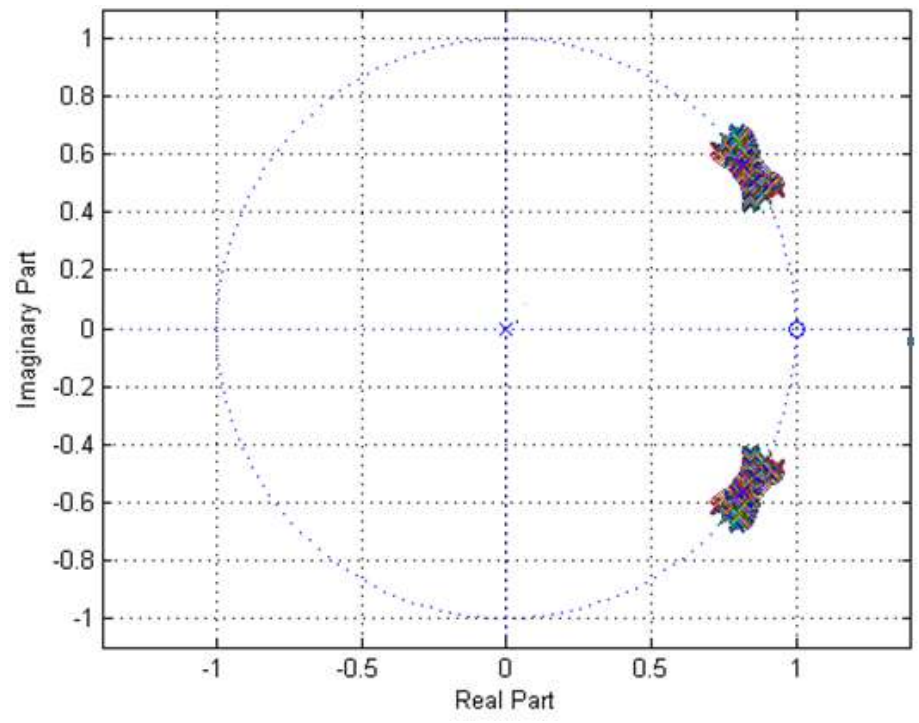

Fig.8.Trajectory of Time Varying poles of first component of Multi Component AM-FM Signal

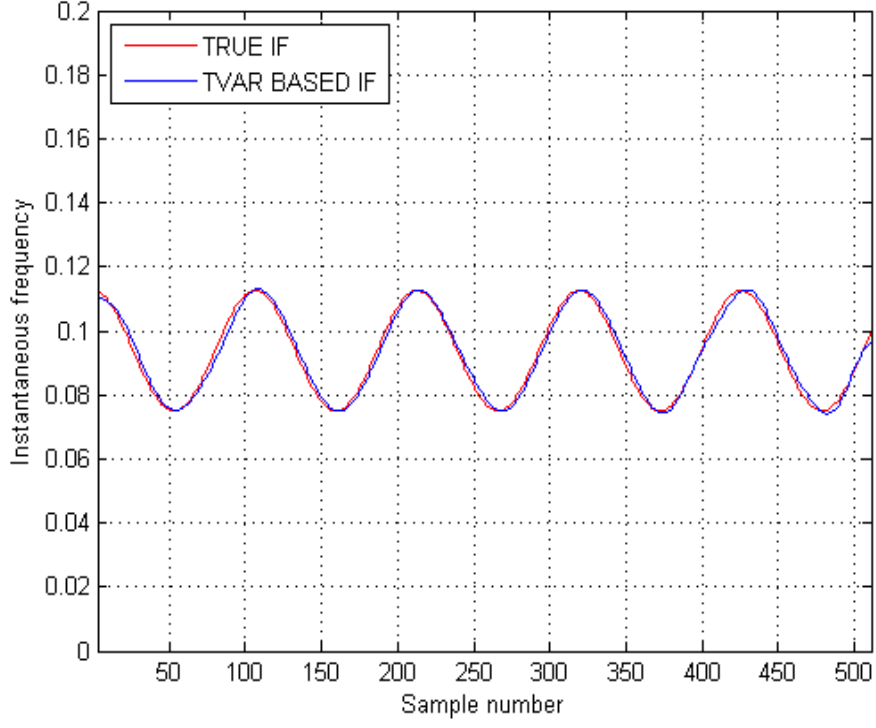

Fig.9.IF Estimation of first component of multi component AM-FM Signal
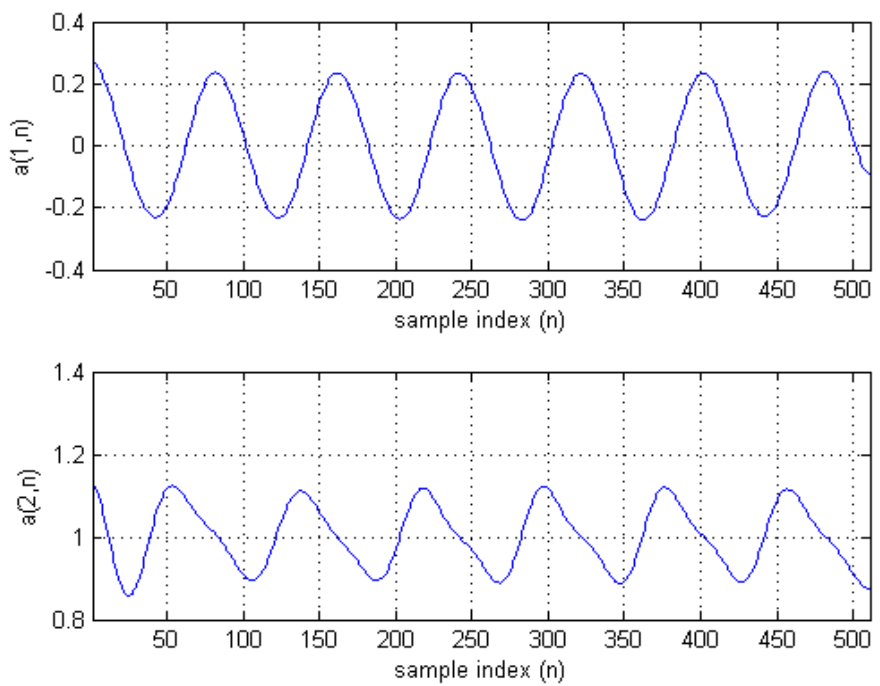

Fig.10.Estimated TVAR Coefficients of second component of Multi-Component AM-FM Signal 


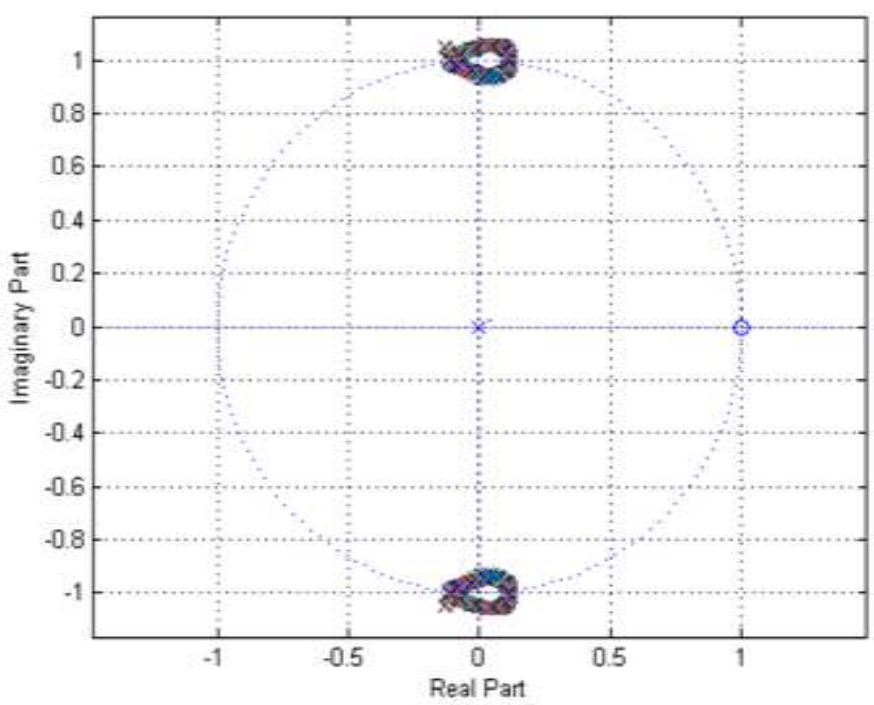

Fig.11.Trajectory of Time Varying poles of second Component of multi component AM-FM Signal

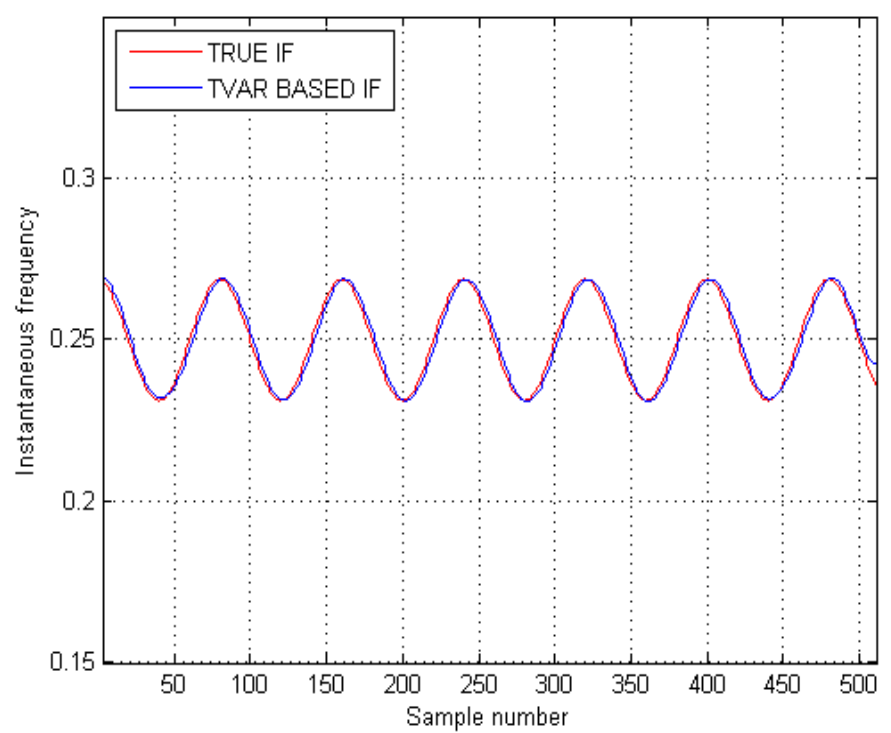

Fig.12.IF Estimation of Second component of Multi -Component AM-FM Signal

2) Multi-component AM-FM signal

$x(t)=\sum_{k=1}^{2} \exp \left(\alpha_{k}\right) \exp \left[j 2 \pi\left(\beta_{k, 3} t^{3}+\beta_{k, 2} t^{2}+\beta_{k, 1} t\right)\right](46)$

The parameters of the first and the second components are[21]

$\alpha_{1}=-2, \beta_{1,1}=10^{3}, \beta_{1,2}=5 \times 10^{3}, \beta_{1,3}=-20 \times 10^{3}$

and $\alpha_{2}=-5, \beta_{2,1}=5 \times 10^{3}, \beta_{2,2}=-3 \times 10^{3}, \beta_{2,3}=0$

The two following IF s are embedded in this AM-FM signal:

$$
\begin{gathered}
f_{1}(t)=-60 t^{2}+10 t+1 k H Z \\
f_{2}(t)=-6 t+5 k H Z
\end{gathered}
$$

The signal in (46) is sampled at $T_{s}=50 \mu$ s intervals, so the sampling frequency is $f_{s}=20 \mathrm{kHz}$. Since the absolute values of the instantaneous frequencies increase over time, a limited span of signal is observed to avoid aliasing. The Algorithm is run over $\mathrm{N}=2,000$ samples.

The plots of the two-component AM-FM signal, the first component, and second component AM-FM signals are shown in Figs.13-15 respectively. The composite signal is expanded into FB expansion and the plot of FB coefficients for the composite signal is shown in Fig.16.The first component of the multi-component signal is separated and reconstructed using 150-400(From Fig.16.) significant nonoverlapping FB coefficients. The reconstructed signal is shown in Fig.17.The second component of the multicomponent signal is separated and reconstructed using 8001100(FromFig.16.) significant non-overlapping FB coefficients. The reconstructed signal is shown in Fig.18.

Each of the two reconstructed AM-FM signals from the clusters of non-overlapped FB coefficients is modeled by TVAR process. By using step 2 in the proposed algorithm we can estimate Instantaneous Frequency (IF) of each reconstructed AM-FM signal.

For separated first component and second component of two component AM-FM signal apply TVAR model with discrete cosine basis. Using Maximum likely hood estimation algorithm we have computed TVAR Model order $\mathrm{p}=2$ and $\mathrm{q}=8$, for first component signal, $\mathrm{p}=2$ and $\mathrm{q}=12$ for second component signal. TVAR coefficients $a_{1, n} a_{2, n}$ for the first component and second component signals are shown in Fig.19.andFig.22.respectively.Trajectory of time varying poles of the first component and second component are shown in Fig.20.and Fig.23.respectively.Fig.21.and Fig.24.shows the IF estimation of first component of two component AM-FM signal and the IF estimation of second component of two component AM-FM signal, respectively .From Fig.21.and Fig.24.we observe that estimated IF is close to true IF. The mean square error (MSE) among the true IF and estimated IF for $n=2,3, \ldots \ldots \ldots, 2000$ is calculated to be $-93.282 \mathrm{~dB}$. and $-86.932 \mathrm{~dB}$ respectively.

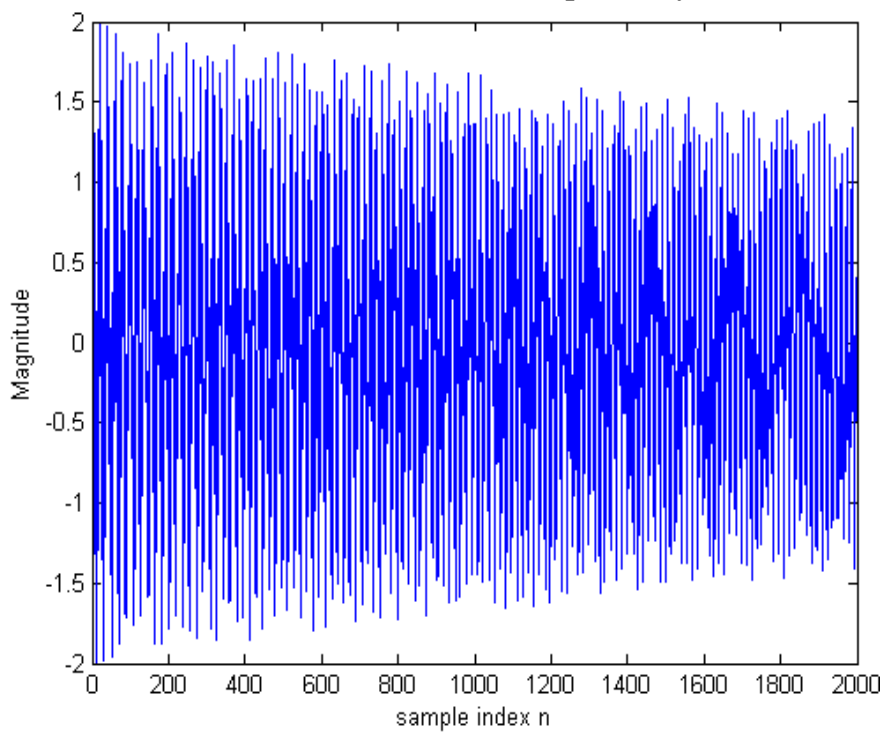

Fig.13.Multi component AM-FM signal 


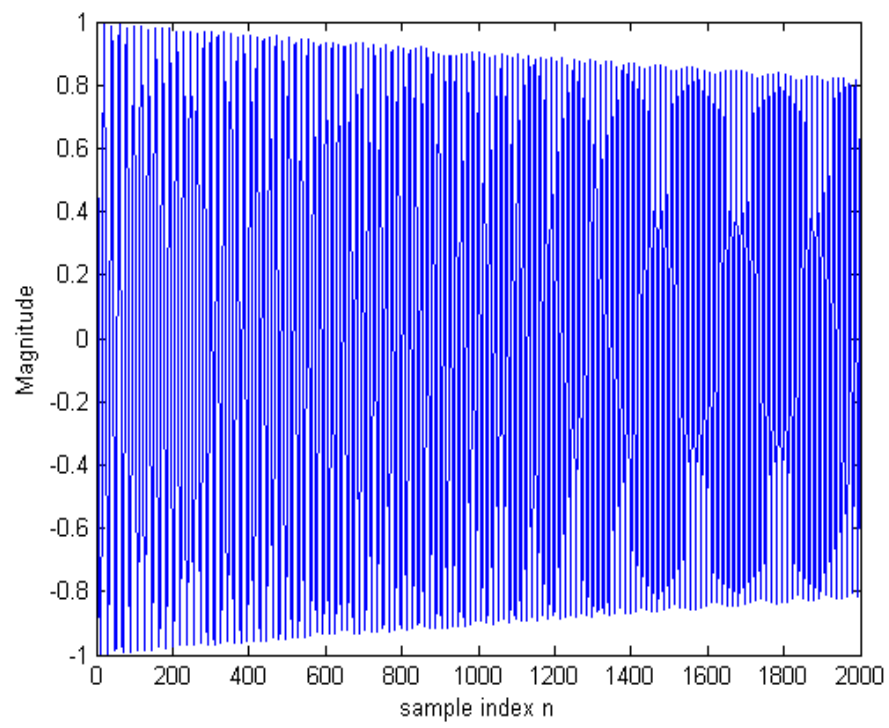

Fig.14.First component of Multi component AM-FM signal

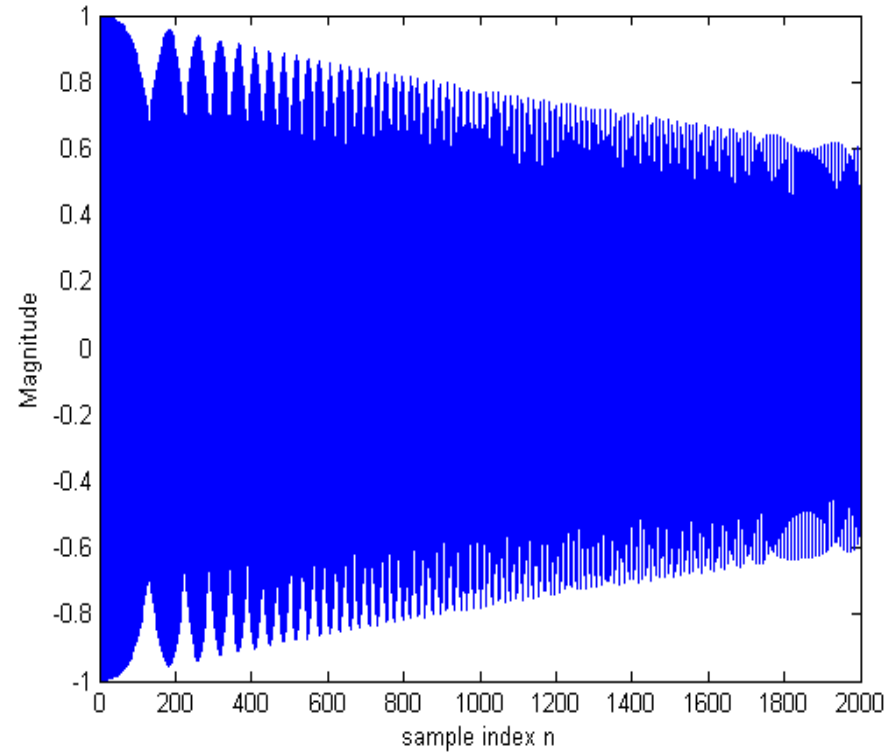

Fig.15.Second component of Multi component AM-FM signal

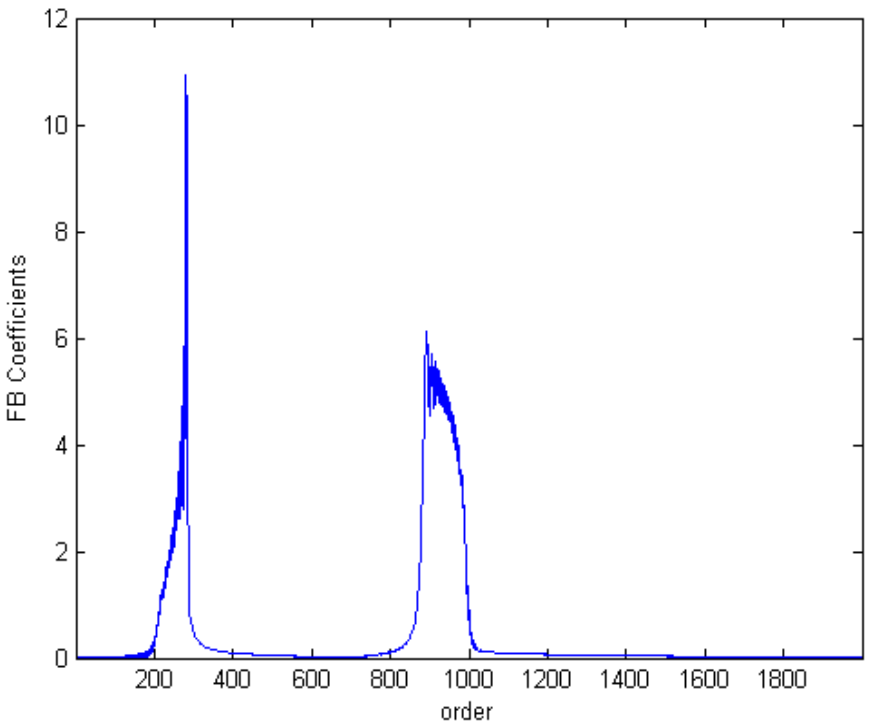

Fig.16.FB coefficients of Multi component AM-FM Signal

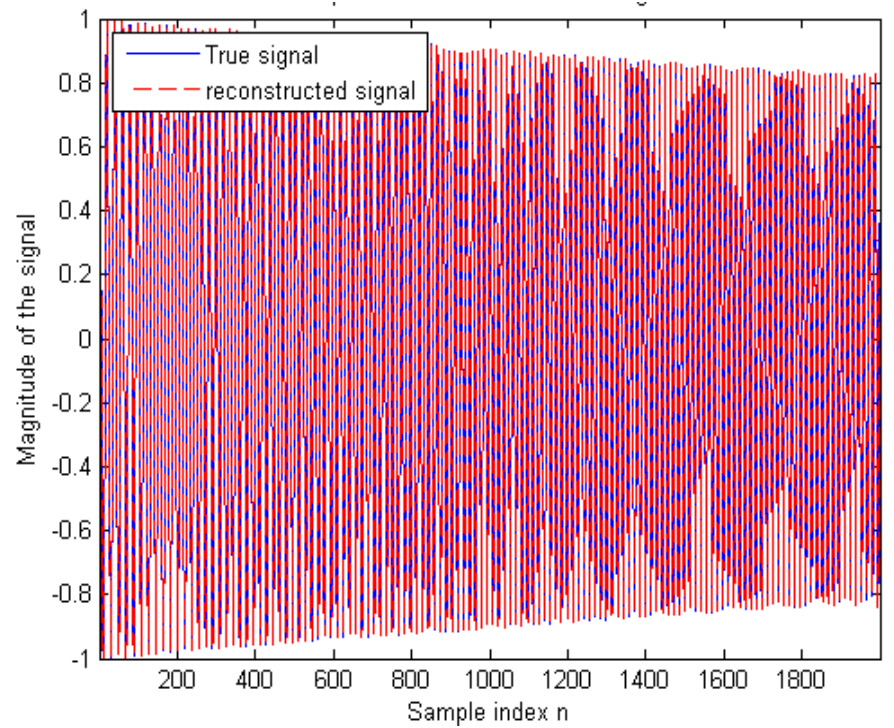

Fig.17.Reconstructed first component of AM-FM signal from FB coefficients

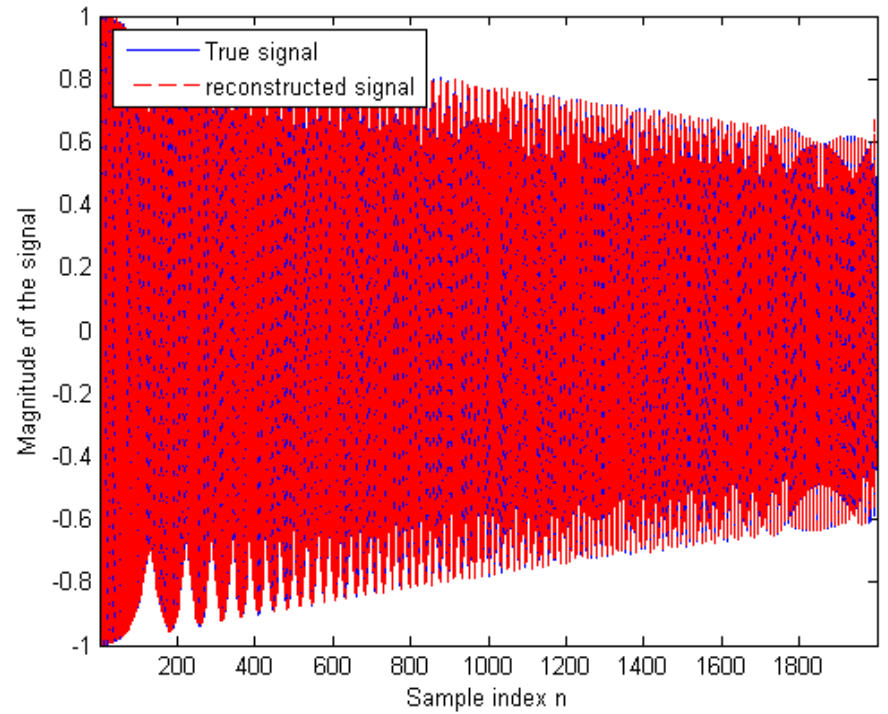

Fig.18.Reconstructed second component of AM-FM signal from FB coefficients
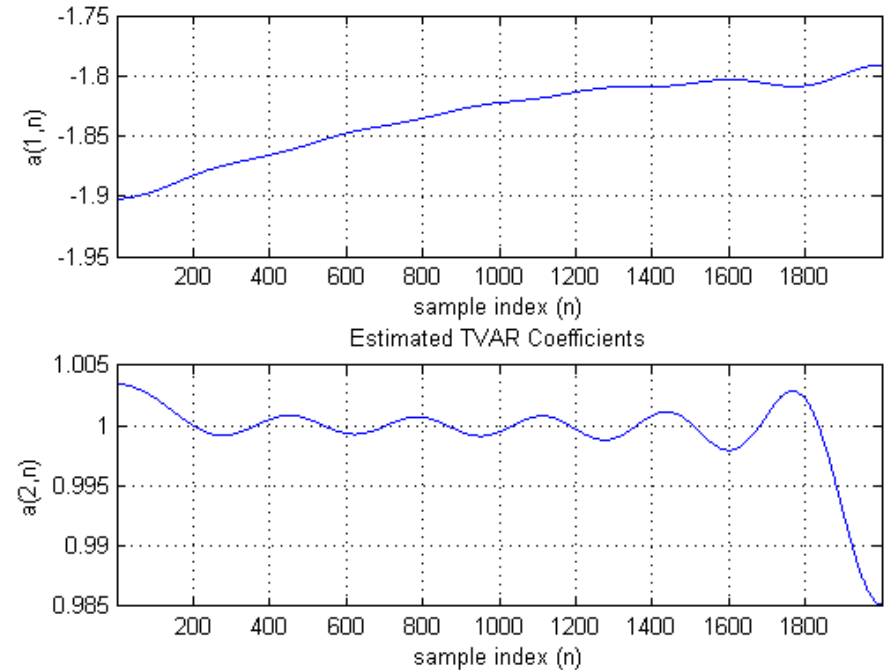

Fig.19.Estimated TVAR coefficients of First component of a multi component AM-FM signal 


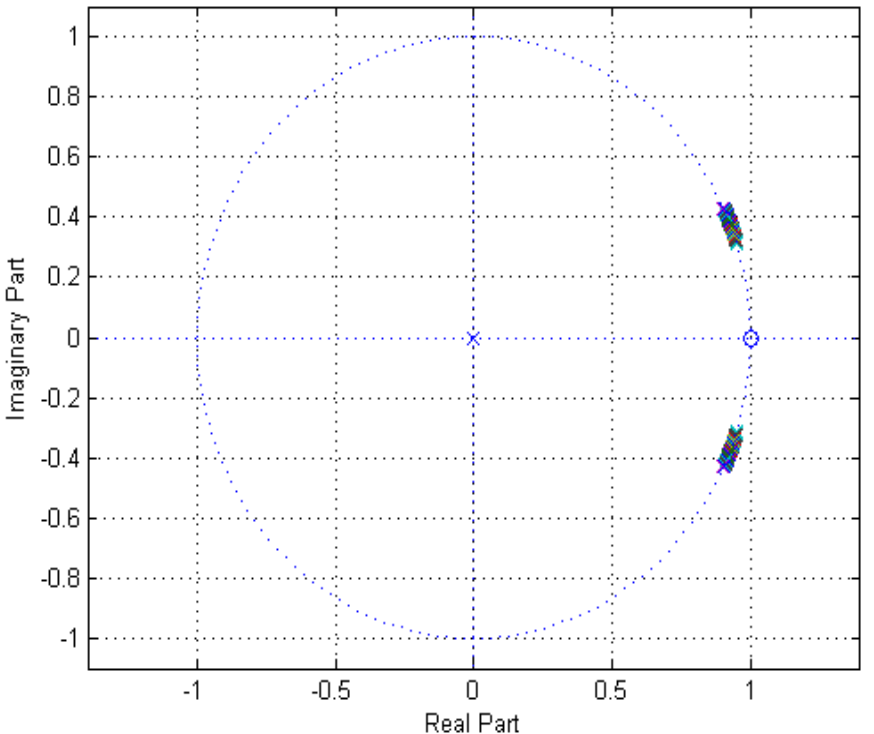

Fig.20.Trajectory of Time Varying of First component of multi component AM-FM signal

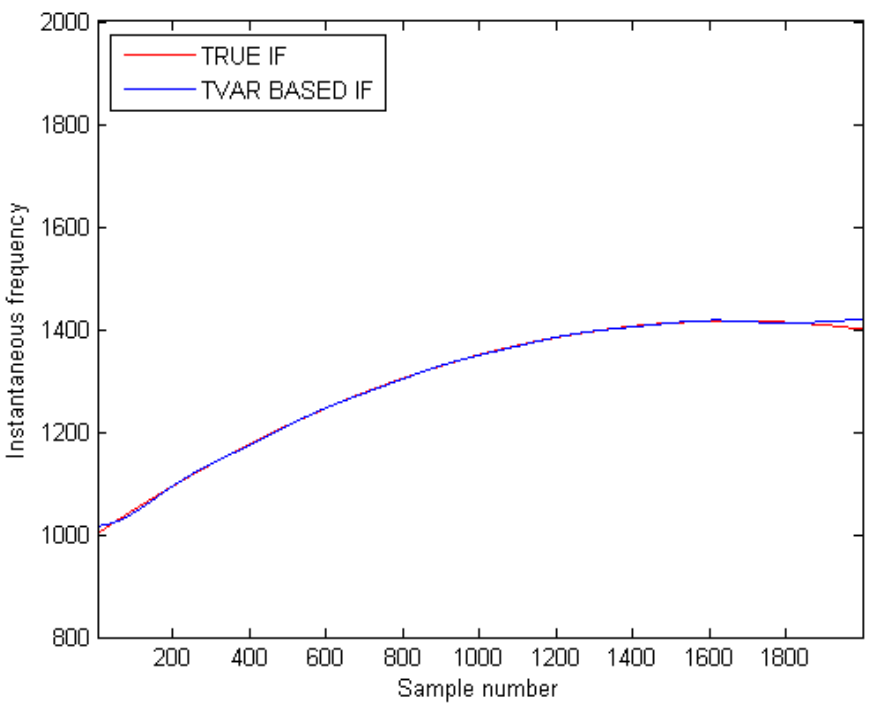

Fig.21.First component IF estimation of Multi component AM-FM signal
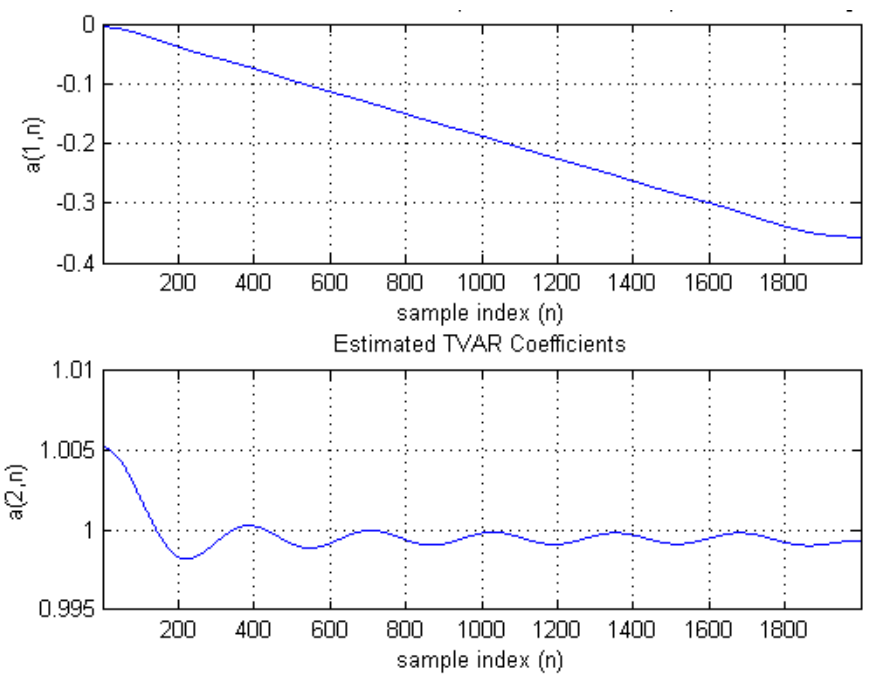

Fig.22.Estimated TVAR coefficients of second component of a multi component AM-FM signal

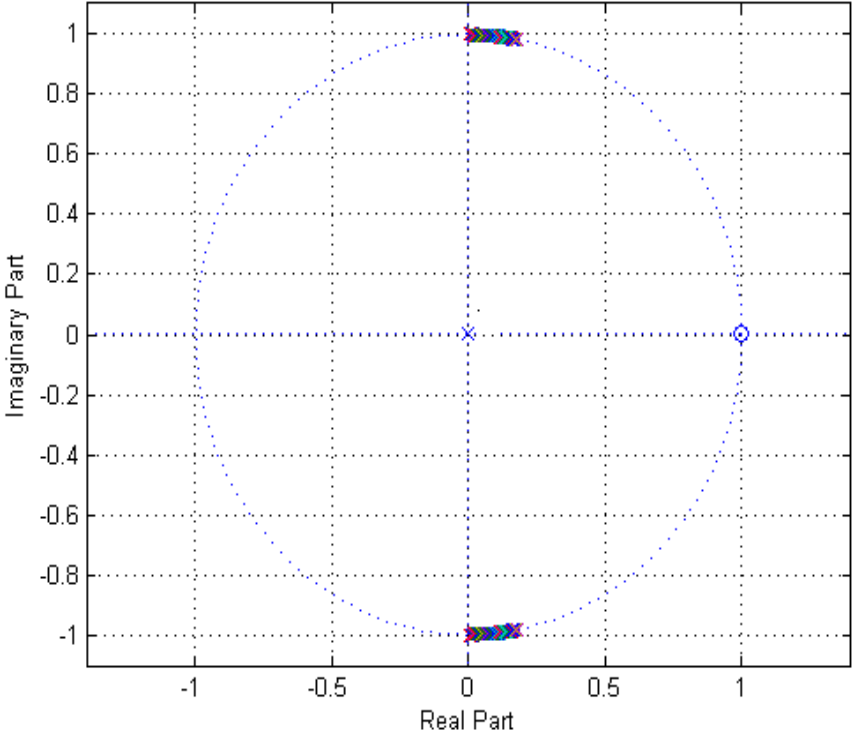

Fig.23.Trajectory of Time Varying of second component of multi component AM-FM signal

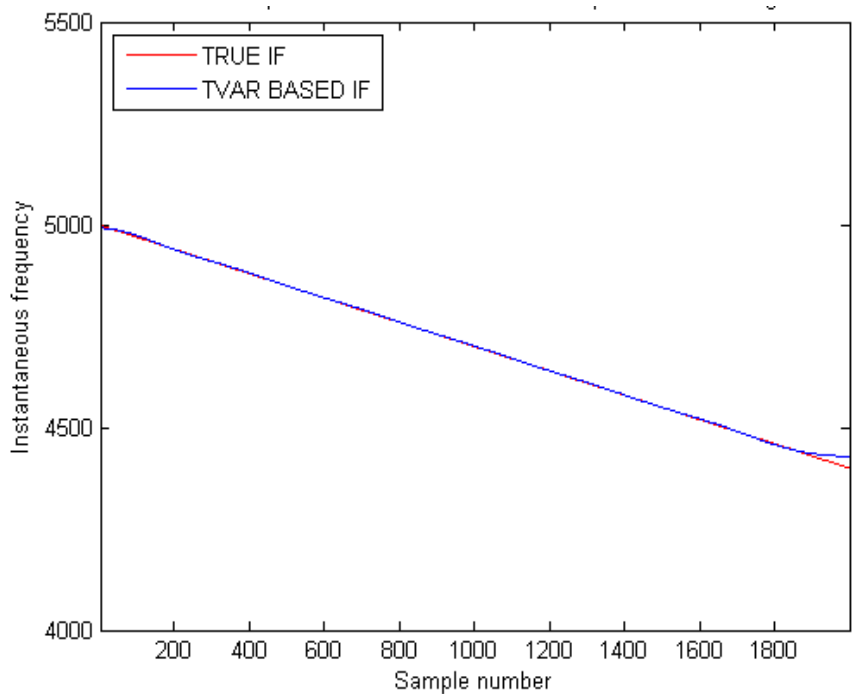

Fig.24.second component IF estimation of Multi component AM-FM signal

\section{CONCLUSIONS}

A new method FB-TVAR, for Instantaneous frequency estimation of multi component AM-FM signals is presented in this paper. The FB expansion decomposes a multicomponent AM-FM signal into a number of mono component signals, and each component signal is modeled using TVAR Model. In this way, the estimation of the model parameters of a multi-component signal is done accurately. The proposed technique does not need any information a priori about the frequency-bands of the multi component AM-FM signals. It is demonstrated that the performance of proposed FB-TVAR method is good for estimation of the frequency functions of an AM-FM signal 


\section{REFERENCES}

[1] B.Boashash,"Time-frequency signal analysis, "in Advances in Spectrum AnalysisandArrayProcessing,(S.Haykin,ed.),Englewood Cliffs, NJ: Prentice Hall, 1991

[2] L.Cohen,"Time-frequency distribution a review, "Proceedings of IEEE, vol.77, no.7, 1989, pp.941-981.

[3] B.Friedlander, J.M.Francos, "Estimation of amplitude and phase parameters of multi-component signals", IEEE Trans on Signal Processing,Vol.51,N0.1,Jan.2003.

[4] L.Rankinea, M.Mesbaha and B.Boashash, "IF estimation for multicomponent signals using image processing techniques in the time frequency domian,"Signalprocessing,Vol.87,2007, pp.1234-1250.

[5] A. Francos, M. Porat, Analysis and synthesis of multi-component signals using positive time- frequency distributions, IEEE Trans. Signal Process. 47 (2) (1999) 493-504.

[6] P.Sircar, M.S. Syali, Complex AM signal model for non-stationary signals, Signal Processing,Vol.53,pp.35-45,1996

[7] P. Sircar, S. Sharma, Complex FM signal model for non-stationary signals, Signal Processing,Vol.53,pp.35-45,1996.

[8] J. Schroeder, Signal processing via Fourier-Bessel series expansion, Digit. Signal Process.3(1993) 112-124.

[9] R.B. Pachori, P. Sircar," Analysis of multi-component AM-FM signals using FB-DESA method" ELSEVIER, Digital Signal processing Vol.20, pp.42-62, 2010

[10] R.B. Pachori, P. Sircar,"Modeling of multi-component AM-FM Signals using FB expansion and linear TVAR process" 16th European Signal Processing Conference(EUSIPCO-2008),Lausnanne, Switzerland.

[11] R.B. Pachori, P. Sircar," Speech analysis using Fourier-Besse expansion and discrete energy Separation algorithm," in: Proc. 12th Digital Signal Processing Workshop and 4thSignal Processing Education Workshop, Wyoming, USA, September 24-27, 2006.

[12] R. B. Pachori and P. Sircar, "EEG signal analysis using FB expansion using FB expansion and second-order linear TVAR process," Signal Processing,Vol.88.pp.415-420,2008.

[13] S. Mukhopadhyay and P. Sircar, "Parametric modeling of non-stationary signals: A unified approach," Signal Processing, Vol. 60, pp. 135-152, 1997.

[14] F.S. Gurgen and C.S. Chen, "Speech enhancement by Fourier-Bessel coefficients of speech and noise," IEE Proceedings, Vol. 137, No. 5, pp. 290-294, Oct 1990.
[15] K. Gopalan and T.R. Anderson, "A Comparison of Speaker Identification Results Using Features Based on Cepstrum and FourierBessel Expansion," IEEE Transaction on Speech and Audio Processing, Vol. 7, No. 3, pp. 289-294, May 1999.

[16] J.J.Rajan and P.J.Rayner, "Generalized Feature Extraction for TimeVarying Autoregressive Model" IEEE Trans on Signal Processing, vol.44, no.10, 1996, pp.2498-2507

[17] F.Kozin and F.Nakajima,"The Order Determination problem for Linear Time-Varying AR Models," IEEE Trans.Automat.contr., Vol 25, no2, 1980, pp.250-257

[18] K.B. Eom, "Analysis of Acoustic Signatures from Moving Vehicles Using Time-Varying Autoregressive Models," Multisignal Systems and Signal Processing, Vol 10, pp 357-378, 1999.

[19] Roshin Kadanna Pally "Implemeentation of instantaneous frequency estimation based on time-varying AR Modeling,"M.S.Thesis,Virginia Tech,April 2009.

[20] Chetana Prakash, Dhananjaya N., and Suryakanth V. Gangashetty, "Analysis of acoustic events in speech signals using Bessel series expansion" International Journal of CircuitsSystem and Signal Processing (Springer), vol. 32, 2013, DOI 0.1007//s00034-013-9596-1

[21] Mohammadli Sebghati, Hamidreza Amindavar, and James A Ritcey, "Basis approach to estimate the instantaneous frequencies in multicomponent AM-FM signals" EURASIP Journal on Audio, Speech, and Music Processing 2014

[22] Yuri I.Abramovich, Nicholas K.Spencer, and Micheal D.E .Turley, "Order Estimation and Discrimination between Stationary and TimeVarying (TVAR) Autoregressive Models" IEEE Transactions on Signal processing, Vol.55, No.6. , June 2007

[23] Y.Pantazis, O.Rosec, and Y.Stylianou, "Adaptive AM-FM signal decomposition with application to speech analysis," IEEE Trans. Audio,Speech,Language Process.,vol.19,no.2,pp.290-300,February 2011

[24] N Khan ,B Boas hash, Instantaneous frequency estimation of multicomponent non-stationary signals using multi view time-frequency distributions based on the adaptive fractional spectrogram, IEEE Signal Process.Lett.20(2),157-160(2013)

[25] G. Ravi Shankar Reddy, Dr.Rameshwar Rao "Non Stationary Signal Prediction using TVAR Model," Proceedings of the IEEE International Conference on Communication and Signal Processing, April 2014,pp.1692-1697. 